Научная статья

Удк 334

DOI https://doi.org/10.24866/VVSU/2073-3984/2021-3/098-104

B. А. Осипов ${ }^{1}$

Люй Гуанхай

Владивостокский государственный университет экономики и сервиса

Владивосток. Россия

\title{
Соотношение понятий конкурентоспособности промышленного предприятия и его продукции
}

\begin{abstract}
Аннотация. Актуальность работы состоит в том, что в ней дается новое понимание связи понятий конкурентоспособности предприятия и изделий, которое можно использовать в системе управления, особенно при маркетинговых исследованиях. Рассмотрены основные процессы формирования функциональной иерархической связи между этими объектами управления. Выявлены пути использования иерархического понимания конкурентоспособности в управлении предприятием с целью повышения его эффективности. Выявлены возможности согласованного управления конкурентоспособностью предприятий различного иерархического уровня. Предлагаются согласованные формулы измерения конкурентоспособности предприятия и его отдельных видов продукции. Выявлена формализованная связь между конкурентоспособностью сложных производственных систем.
\end{abstract}

Ключевые слова: конкурентоспособность изделия, конкурентоспособность предприятия, иерархия и связь понятий, методы измерения, пищевая промышленность.

V.A. Osipov

Lu Guang hai

Vladivostok State University of Economics and Service

Vladivostok. Russia

\section{Correlation of the concepts of competitiveness of an industrial enterprise and its products}

\footnotetext{
Abstract. The relevance of the work consists in the fact that a new understanding of the relationship between the concepts of enterprise competitiveness and products is given, which can be used in the management system, especially in marketing research. The main processes of forming a functional hierarchical relationship between these management objects

${ }^{1}$ Осипов Виктор Алексеевич - д-р экон. наук, профессор, профессор кафедры экономики и управления. ORCID: https://orcid.org/0000-0002-0888-4889; e-mail:professorosipov17@mail.ru 2 Люй Гуанхай - аспирант кафедры экономики и управления; e-mail: 1215111712@qq.com 98
} 
are considered. The possibilities of coordinated management of competitiveness of enterprises of various hierarchical levels are revealed. The agreed formulas for measuring the competitiveness of an enterprise and its individual types of products are proposed. A formalized relationship between the competitiveness of complex production systems is revealed.

Keywords: product competitiveness, enterprise competitiveness, hierarchy and relationship of concepts, measurement methods, food industry.

Актуальность работы состоит в том, что для промышленных предприятий до сих пор не предложены адекватные механизмы решения известной теоретической проблемы: «что производить» - это принятие решений о том, какие блага, какого качества, в каком количестве должны быть произведены; «как производить» - это принятие решений о том, с помощью каких ресурсов, с помощью каких технологий будут произведены блага; «для кого производить» - это проблема, связанная с распределением благ: кому достанутся произведенные блага.

Сейчас понятно, что конкуренция в промышленности является движущей силой развития общества, повышения качества товаров и уровня жизни населения. Цели, стоящие перед конкретным предприятием, определяют, какие результаты и когда должны быть достигнуты. Они фактически дают картину будущего потребления продукции промышленного производства.

Исследование методов оценки конкурентоспособности продукции показало отсутствие единого методического подхода к этому вопросу, как в России, так и за рубежом [3].

Развивая предложенные в литературе идеи и методы по формированию показателей конкурентоспособности, будем опираться на понимание конкурентоспособности продукции как удаленной формы эффективности производства (предприятия), которое создало эту продукцию. Предлагаем конкурентоспособность продукции представлять как форму эффективности данного производства, реализуемую у потребителя:

$$
K=\frac{V}{C+D},
$$

где $K$ - конкурентоспособность изделия (продукта);

$V$ - потребительская ценность изделия (полезный эффект у потребителя, качество);

$C$ - затраты на приобретение изделия (цена продажи);

$D$ - затраты потребителя по использованию изделия.

В литературе отмечается, что главным препятствием для использования этой формулы является измерение потребительской ценности продукта $(V)$. Предлагаются разные подходы к измерению потребительской ценности, как на основе экспертных оценок, так и аналитических формул [6]. Например, для пищевой промышленности полезный эффект у потребителя количественно может быть измерен содержанием полезных веществ для организма человека (белки, жиры, углеводы), которые содержатся в единице продукта. Используются сложные подходы и формулы, которые фактически уводят от сущности конкурентоспособности продукции [4]. Потребительская ценность, на 
наш взгляд, должна отражать потенциальный (при планировании) и фактический эффект от потребления продукта.

Знаменатель в предлагаемой формуле отражает суммарные затраты по приобретению и потреблению продуктов. Вторая составляющая для определения в пищевой промышленности обычно не используется, так как считается, что потребление пищи - это разовый акт. Но для отраслей с длительным потреблением изделий (машиностроение) затраты по эксплуатации являются существенным фактором, влияющим на конкурентоспособность изделий. С теоретической точки зрения эти затраты следует рассматривать как продолжение процесса производства продукции до их полного потребления.

Можно отметить, что при определении конкурентоспособности товара важно знать удельные затраты на единицу потребительского эффекта от изделия. Обратная величина показателя конкурентоспособности покажет удельные затраты в расчете на единицу потребительского эффекта. Даже при низком качестве товар будет продан, если задать ему более низкую цену. Конкуренция должна опираться как на потребительские эффекты изделий, так и на затратные характеристики.

В формуле (1) наиболее сложным является определение полезного эффекта изделия у потребителя. Широко применяются при этом методы с использованием экспертных оценок потребительских параметров изделий. При определении набора оцениваемых параметров выбирают те, которые представляют существенный интерес для потребителей данного рынка. Часть параметров характеризуют потребительские свойства товара (или потребительскую ценность). К ним относятся важнейшие функции товара и их основные характеристики, соответствие стандартам безопасности, содержание белков, жиров, углеводов, органолептические, эстетические и другие параметры.

Изучение конкурентоспособности собственного товара, представленного на рынке, должно вестись производителем непрерывно. Следует учитывать, что новый товар при выходе на рынок сразу (по мере возникновения новых продуктов у конкурентов) начинает в той или иной мере терять свою конкурентоспособность, поэтому конкурентоспособность новых товаров должна быть опережающей и, возможно, долговременной.

Сложным и не решенным в литературе является вопрос функциональной связи конкурентоспособности изделия и прибыли от его реализации [1]. Внутриотраслевая конкуренция предприятий основывается на сопоставлении «извлекаемой» с рынка прибыли. Считается, что конкурентоспособность продукции и конкурентоспособность предприятия-производителя соотносятся как часть и целое. Однако не предложено какое-либо аналитическое описание этой связи. Имеются попытки описать конкурентоспособность предприятия на основе корреляционных методов [5]. Возможности предприятия конкурировать на конкретном рынке зависят от конкурентоспособности продукции и совокупности социально-экономических и организационных факторов и методов деятельности предприятия [2].

Понятно, что только конкурентоспособное изделие реализуется с прибылью. Конкурентоспособность - это главное ограничение по товару, которое приносит прибыль, то есть по своей экономической сущности конкурентоспособность предприятия представляет собой более широкую категорию, чем конкуренто- 
способность продукции. Но должна быть выявлена и более четкая связь между этими категориями.

Рассмотрим возможность определения иерархии конкурентоспособности. Вернемся к формуле конкурентоспособности изделия как к частному от деления потребительской ценности единицы товара к затратам на производство и потребление изделия. Это выражение показывает удельную отдачу затрат на производство и потребление продукции, приходящиеся на единицу потребительского эффекта. При этом суммирование показателей конкурентоспособности как удельных показателей невозможно или ничего не дает для понимания перехода от конкурентоспособности изделия к конкурентоспособности предприятия. Но если суммировать отдельно числители показателей конкурентоспособности по всем произведённым изделиям предприятия за какой-либо период времени (год, месяц, день), то эта величина покажет суммарный или интегральный потребительский эффект (ИПЭ), реализуемый от производственной деятельности предприятия. В случае однопродуктового производства формула может выглядеть следующим образом:

$$
S_{i}=\sum_{i=1}^{n} V_{i},
$$

где $S_{i}-$ интегральный (суммарный) потребительский эффект от произведённой продукции ( $i$-го вида изделия);

$i$ - вид производимого изделия;

$n$ - количество произведённых предприятием и потребленных за данный период изделий.

В случае многономенклатурного производства эта формула преобразуется в следующий вид:

$$
S_{i j}=\sum_{i=1}^{n} \sum_{j=1}^{m} V_{i j}
$$

где $j$ - количество видов производимой продукции на предприятии;

$m$ - количество видов продукции, выпускаемых на предприятии (номенклатура).

Формула (3) отражает величину покрываемой на данном рынке потребности или суммарного потребительского эффекта от продукции заданного ассортимента, то есть от производства $i$-го количества изделий $j$-го наименования.

Данная величина отражает цель деятельности предприятия, измеряемую за календарный период. В системе управления она должна ставиться как нормативный показатель, который должен быть выполнен. Именно этим показателем, на наш взгляд, должна также измеряться доля рынка товара, производимого данным предприятием.

Если эту величину отнести на суммарные затраты по производству и потреблению продукции, полученная величина будет отражать конкурентоспособность данного производства в заданный период. Это можно выразить в виде формулы, представленной ниже:

$$
P_{i}=\frac{S_{i}}{C_{i}+D_{i}}
$$

где $P_{i}$ - конкурентоспособность предприятия по производству $i$-го продукта;

$S_{i}$ - интегральный потребительский эффект от произведённой продукции ( $i$-го вида изделия); 
$C_{i}$ - затраты по изготовлению $i$-го вида изделий за календарный период;

$D_{i}$ - суммарные затраты по потреблению $i$-го вида изделий совокупным потребителем.

Поскольку предприятия пищевой промышленности чаще всего бывают многономенклатурными, то конкурентоспособность их должна измеряться по формуле

$$
P_{i j}=\frac{S_{i j}}{C_{i j}+D_{i j}},
$$

где $S_{i j}$ - интегральный потребительский эффект от потребления $i$-го количества изделий $j$-го наименования;

$C_{i j}$ - затраты по изготовлению і-го количества изделий $j$-го наименования;

$D_{i j}$ - затраты по потреблению і-го количества изделий $j$-го наименования.

Всегда крайне сложно в многономенклатурном производстве выделить затраты, приходящиеся на изучаемую совокупность изделий. Отсюда следует, что необходимо обратиться к опыту определения калькуляций одного изделия и рассматривать общие затраты производства как суммы затрат по калькуляциям изготовленных изделий. Аналогично следует измерять и затраты потребления. Тогда формула конкурентоспособности предприятия будет представлена в виде

$$
P_{i j}=\frac{S_{i j}}{\sum_{i=1}^{n} \sum_{i=1}^{m} C_{i j}+\sum_{i=1}^{n} \sum_{k=1}^{l} D_{i k}},
$$

где $S_{i j}$ - интегральный потребительский эффект от потребления $i$-го количества изделий $j$-го наименования;

$C_{i j}$ - сумма затрат по калькуляциям изготовления $i$-го количества изделий $j$-го наименования;

$D_{i k}$ - суммарные затраты по потреблению $i$-го количества изделий у $k$-го покупателя;

$l$ - количество потребителей, купивших $i$-й продукт.

Эту формулу предлагается рассматривать в качестве показателя эффективности производственной системы в целом. В числителе отражается потребительский эффект, получаемый от всей продукции предприятия за календарный период, а в знаменателе суммарные затраты по всем калькуляциям отражают затраты на производство за тот же период. На наш взгляд, это и есть конкретная формула связи конкурентоспособности изделий и конкурентоспособности предприятий.

Когда мы переходим на более высокий иерархический уровень определения конкурентоспособности - отраслевой, региональный или даже государственный, то конкурентоспособность экономической системы следует определять как степень удовлетворения потребности в промышленной (пищевой) продукции населения, то есть на основе числителя этой формулы. В случае регионального или государственного уровня определения конкурентоспособности экономики следует опираться на понятие «потребительская корзина» [7]. Это стандартизированный набор товаров и услуг, характеризующий 
В.А. Осипов, Люй Гуанхай. Соотношение понятий конкурентоспособности ...

уровень и структуру потребления человека или семьи. В Российской Федерации ее используют только для определения динамики цен - инфляции. При этом второе значение потребительской корзины как целевого минимального стандарта потребления благ выпало из системы управления. Хотя именно в таком понимании её используют, например, в Казахстане и других странах. При достижении стандарта ставится следующая задача снижения её стоимости или увеличения набора продуктов потребительской корзины (опыт США, Франции, ФРГ).

Вывод: повышение конкурентоспособности предприятия тождественно повышению экономической эффективности деятельности хозяйствующих субъектов, а сама конкурентоспособность - это одна из форм проявления эффективности с учетом реального потребления продукции.

Потребность в каком-либо продукте можно рассматривать как осознание человеком (субъектом хозяйствования, лицом, принимающим решение) на основе маркетинговых исследований потери от неполучения благ, потребительских ценностей. При этом главным условием возникновения потребности является упреждающее наличие ресурсов для получения потребительского эффекта. Условно говоря, мы можем удовлетворить потребности сегодняшнего дня только завтра, через некоторый период времени производства: от осознания потребности до ее реализации. Следовательно, чем скорее удовлетворяются потребности, тем выше эффективность производственной системы.

В любом случае объективное определение конкурентоспособности промышленного производства на различных иерархических уровнях будет способствовать объективности маркетинговых исследований и росту его эффективности.

1. Ворожбит О. Ю., Осипов В. А., Тонких А.И. Конкурентоспособность экономических систем. - Владивосток: Изд-во ВГУЭС, 2011. - 139 с.

2. Ворожбит О. Ю., Шашло Н. В., Люй Гуанхай. Эволюционная трансформация понятийно-категориального аппарата теорий предпринимательства в условиях конкуренции // Азимут научных исследований: экономика и управление. - 2018. - Т. 7, № 1 (22). - C. 261-265.

3. Воронов Д. С. Обзор существующих методов оценки конкурентоспособности предприятия. - Текст: электронный / Уральский федеральный университет. - URL: http://vds1234.ru/?31.html. (дата обращения: 13.01.2017).

4. Парамонова Т. Н. Методика расчета конкурентоспособности товара. - Текст: электронный. - URL: http://www.elitarium.ru/konkurentosposobnost-tovara-ocenka-kachestvoprodukcijasravnenie-metodika/ (дата обращения: 12.01.2017).

5. Сартбаев С.Е. Методические рекомендации по анализу показателей конкурентоспособности предприятия. - Москва: ИНФРА-М, 2017. - 176 с.

6. Тарануха Ю.В. Конкуренция и конкурентоспособность. - Москва: РУСАЙНС, 2020. $334 \mathrm{c}$.

7. Федеральный закон Российской Федерации от 3 декабря 2012 г. № 227-Ф3 «О потребительской корзине в целом по Российской Федерации» 


\section{Транслитерация}

1. Vorozhbit O. Yu., Osipov V.A., Tonkih A. I. Konkurentosposobnost' ekonomicheskih sistem. - Vladivostok: Izd-vo VGUES, 2011. - 139 s.

2. Vorozhbit O. Yu., Shashlo N. V., Lyuj Guanhaj. Evolyucionnaya transformaciya ponyatijno-kategorial'nogo apparata teorij predprinimatel'stva v usloviyah konkurencii // Azimut nauchnyh issledovanij: ekonomika i upravlenie. - 2018. - T. 7, № 1 (22). - S. 261-265.

3. Voronov D.S. Obzor sushchestvuyushchih metodov ocenki konkurentosposobnosti predpriyatiya. - Tekst: elektronnyj / Ural'skij federal'nyj universitet. - URL: http://vds1234.ru/?31.html. (data obrashcheniya: 13.01.2017).

4. Paramonova T.N. Metodika rascheta konkurentosposobnosti tovara. - Tekst: elektronnyj. URL: $\quad$ http://www.elitarium.ru/konkurentosposobnost-tovara-ocenka-kachestvoprodukcijasravnenie-metodika/ (data obrashcheniya: 12.01.2017).

5. Sartbaev S. E. Metodicheskie rekomendacii po analizu pokazatelej konkurentosposobnosti predpriyatiya. - Moskva: INFRA-M, 2017. - $176 \mathrm{~s}$.

6. Taranuha Yu. V. Konkurenciya i konkurentosposobnost'. - Moskva: RUSAJNS, 2020. $334 \mathrm{~s}$.

7. Federal'nyj zakon Rossijskoj Federacii ot 3 dekabrya 2012 g. № 227-FZ «O potrebitel'skoj korzine v celom po Rossijskoj Federacii»

(C) В. А. Осипов, 2021

( ) Люй Гуанхай, 2021

Для цитирования: Осипов В.А., Люй Гуанхай. Соотношение понятий конкурентоспособности промышленного предприятия и его продукции // Территория новых возможностей. Вестник Владивостокского государственного университета экономики и сервиса. - 2021. - Т. 13, № 4. - С. 98-104.

For citation: Osipov A.V., Lu Guang hai. Correlation of the concepts of competitiveness of an industrial enterprise and its products, The Territory of New Opportunities. The Herald of Vladivostok State University of Economics and Service, 2021, Vol. 13, № 4, pp. 98-104.

DOI https://doi.org/10.24866/VVSU/2073-3984/2021-4/098-104

$\begin{array}{lll}\text { Дата поступления: } & \text { Одобрена после рецензирования: } & \text { Принята к публикации: } \\ 06.10 .2021 & 09.11 .2021 & 15.11 .2021\end{array}$

